\title{
Des castes en Europe et des Roms au sein de l'humanité
}

\section{Rada Ivekovic}

\section{(2) OpenEdition \\ 1 Journals}

\section{Édition électronique}

URL : http://journals.openedition.org/conflits/18159

DOI : 10.4000/conflits.18159

ISSN : $1777-5345$

Éditeur :

CCLS - Centre d'études sur les conflits lilberté et sécurité, L'Harmattan

\section{Édition imprimée}

Date de publication : 5 septembre 2011

Pagination : 223-227

ISBN : 978-2-296-56086-4

ISSN : 1157-996X

\section{Référence électronique}

Rada Ivekovic, "Des castes en Europe et des Roms au sein de l'humanité ", Cultures \& Conflits [En ligne], 81-82 | Printemps/Été 2011, mis en ligne le 05 septembre 2012, consulté le 30 mars 2021. URL : http://journals.openedition.org/conflits/18159; DOI : https://doi.org/10.4000/conflits.18159 


\section{Des castes en Europe et des Roms au sein de I'humanité}

\section{Rada IVEKOVIC}

Rada Ivekovic est philosophe et indianiste, universitaire en France et ancienne $d u$ Collège international de philosophie. Elle est membre $d u$ reseau de recherche TERRA et de Transeuropéennes. Parmi ses publications figurent Le sexe de la nation (Paris, Léo Scheer 2003), Dame Nation. Nation et différence des sexes (Ravenna, Longo Editore 2003). Sortira prochainement Paradoxes de la souveraineté. Le charme discret des bouddhismes (Paris, Klincksieck).

Tos catégories sociologiques ont une histoire. Elles sont souvent norma1 tives et stigmatisantes. Après les évictions brutales d'Italie depuis plusieurs années, depuis les traitements douteux qui leur sont administrés en Slovaquie ou en Roumanie en guise de remède, depuis leur déportation de France à l'été 2010, nous n'entendons plus beaucoup parler des Roms. Certes, il y a eu des protestations et des pétitions. Les Roms ne suscitent aucun intérêt public à plus grande échelle pour les mêmes raisons qui font qu'ils sont ainsi traités. Il y a des réactions scandalisées ou « alibis » sur le moment, mais personne ne pense sérieusement à résoudre ce problème européen, car il est constitutif de nos sociétés. Il faudra qu'ils se mobilisent par eux-mêmes. Or, ils sont dispersés, et leurs associations sont «culturalisées », " misérabilisées » et dépolitisées. Ils sont bien au-delà des « lignes abyssales » comme dirait Boaventura de Sousa Santos ${ }^{1}$.

L'Europe est fière de son humanisme, mais n'est pas capable d'embrasser tout le monde en lui. Surgissent toujours de nouvelles exceptions à la règle de l'universalité. C'est d'ailleurs ainsi que l'universel fonctionne, puisqu'une prétention universelle a nécessairement une origine particulière. Après les migrants et les musulmans en général (par une « universalisation » abusive des islamistes), les Tsiganes en sont le dernier exemple en Europe. En effet, les

1. De Sousa Santos B., "Beyond Abyssal Thinking: From Global Lines to Ecologies of Knowledge”, Review, XXX, 1, 2007, http://www.ces.uc.pt/bss/documentos/ AbyssalThinking.pdf et Eurozine, http://www.eurozine.com/pdf/2007-06-29-santos-en.pdf. Page consultée le 26 mai 2011. 
Roms, population aux confins aussi bien des États que du droit et de l'humanisme, séjournent en un limbe véritablement européen. Ils ne sont ni dehors ni dedans. Leur cas est extraordinaire et montre toute l'hypocrisie et les limites de l'humanisme européen. Car les Roms sont en Europe exactement comme une caste en Inde, et en particulier comme la caste des « intouchables » (aujourd'hui les dalit). Mais le concept européen de caste est un concept normatif de sociologie européenne pour l'Inde. Il ne correspond à rien directement ni sans reste dans la culture et l'expérience «indiennes». Il ne doit par conséquent jamais être utilisé en Europe (cette prescription est sous-entendue et forte), et s'applique à des contrées lointaines dans le temps et dans l'espace, comme les termes « ethnie », « tribu », etc. Il est parmi ces concepts qui ne s'appliquent qu'aux autres. Ce n'est pas pour dire que l'Inde est une société égalitaire, mais c'est là une autre question.

Des continuités instrumentales, mais réelles, c'est-à-dire historiquement productives, ont été établies entre des systèmes d'éducation locaux et leurs hiérarchies sociales correspondantes, avec les valeurs et sciences historiquement coloniales. Celles-ci avaient pour but de délester les savoirs occidentaux de toute responsabilité politique tout en leur assignant le rôle de remédier à l'« irrationalité » pré-moderne sur place. Systématiquement, des groupes sociaux ont été dépolitisés ${ }^{2}$ dans la construction même du concept idéologisé de « caste ». Ce concept est vidé de ses éléments de classe sociale, d'inégalités politiques et économiques; il est réduit aux mœurs, aux coutumes et à l'éthique. On dépolitisait ainsi la société indienne, mais on disculpait l'occupant de toute responsabilité envers elle. On maintenait ainsi la souveraineté nationale impériale, exprimée dans le droit de la nation colonisatrice à se déporter en dehors d'elle-même sans en accorder la réciprocité aux colonisés. Ce «droit » repose sur un privilège sous-entendu et primaire que l'on s'octroie, celui de la propriété : il est collectivement porté aux colonies. On esthétisait, ethnicisait ou mythologisait alors au maximum l'Inde, comme tous les pays coloniaux. On la voyait par une grille exclusivement religieuse, on y réduisait le social et le politique à une sphère morale.

Le caractère normatif et dé historisé d'un concept venant de la sociologie occidentale a certainement contribué à le figer et a même eu des effets de retour en Inde. On s'est gardé d'accorder ce terme à des configurations sociales européennes, même si certaines, et en particulier aujourd'hui le traitement des Roms, correspondent tout à fait à l'idée qu'on s'en fait : au sujet de ces derniers, comme pour les «castes », intervient par exemple la barrière du travail physique, celle de la pollution imaginaire et celle du symbolique. Mais

2. De Bouglé C., Essai sur le régime des castes, Paris, PUF, 1908 (aussi sur http://classiques.uqac.ca/classiques/bougle_celestin/essai_regime_des_castes/essai_regime_c astes.html, page consultée le 21 janvier 2011) à Dumont L., Homo hierarchicus. Essai sur le système des castes, Paris, Gallimard, 1969. Les théoriciens de la « caste " dépolitisent ce concept et la société à laquelle ils l'assignent. Max Weber est plus prudent et visionnaire en utilisant le concept de Stand: Hindonisme et bouddhisme, Paris, Flammarion, 2003. 
le terme est proscrit d'utilisation pour l'Europe et apparaît rarement pour d'autres régions que l'Inde, bien que parfois des contrées «éloignées » en Afrique, en Caraibe française ${ }^{3}$ ou au Japon (allusions vagues concernant le statut de la population autochtone ou des Coréens) - c'est-à-dire le traitement des indigènes - soient citées pour comparaison ${ }^{4}$. Ceci ne fait en rien disparâ̂tre la vérité sociale, économique et politique de l'impitoyable discrimination par groupe de naissance que l'on retrouve en Inde, comme c'est le cas en Europe au sujet des Tsiganes. Avec tout ceci, dans la construction de connaissances sur le pays éloigné, le regard « touristique » est complété par le regard «salvateur» occidental.

Le terme de caste est donc rarement utilisé pour d'autres pays. Pourtant, la même idée de souillure physique et de dégout est commune aux deux (les dalit et les Roms, dans leurs environnements dominants respectifs). Une différence consiste en ce que les dalit ont réussi à s'afficher sur la scène politique de l'Inde, un espace de politique représentative extrêmement vivant (plus vivant que celui de la sphère politique désabusée, résignée ou blasée de l'Europe). Alors que tout le monde l'aurait cru impossible il y a encore quelques décennies, les dalit sont devenus politiquement visibles et puissants comme groupe (et comme sujet collectif), alors que ce n'est pas encore véritablement le cas des Roms, fragmentés par les frontières et les politiques nationales. Ils avaient une certaine représentativité formelle en ancienne Yougoslavie par exemple, mais leur condition a empiré depuis dans ces régions et ne s'est pas globalement améliorée en Europe non plus. Dans les guerres yougoslaves des années quatre-vingt-dix du XXe siècle, les Roms furent les seuls à ne pas revendiquer de territoires. C'est sans doute ce qui les a condamnés.

Ils ne correspondent pas non plus au seul double rejet que Balibar 5 détecte en Europe. Leur excrétion par la société ne peut être réduite au seul refus du travail physique au regard du travail intellectuel, estimé plus « chic », puisque ce refus ne concerne pas les femmes en Europe, qui continuent à faire le travail physique ménager même quand elles sont des intellectuelles et que leurs compagnons le sont également. Les Roms sont écartés par un préjugé

3. Bruneteaux P. et Zander U., "Quel colonialisme dans la France d'outre-mer ? ", Appel à contributions pour "Asylon(s)", sur http://www.reseau-terra.eu/article1081.html, page consultée le 26 mai 2011.

4. Bujra A., The Politics of Stratification, Oxford University Press, 1971. Cet ouvrage décrit la société sud-yéménite comme étant traversée par le "castisme », bien qu'il refuse aussi bien le terme de caste que de classe. Dans les groupes tels que décrits, «Attas » correspond bien, dans la série des varna, au stéréotype de kšatriya, «Basahl » à celui de brahmane, « Masakin » à celui de vaišya, et « Hirthan » à baniya, alors que « Akhdam » correspond à śúdra.

5. Balibar É., La proposition de l'égaliberté. Essais politiques 1989-2009, Paris, PUF, 2010, p. 80. Balibar voit différents types d'exclusion ou d'inégalité en Europe : celui basé sur la différence entre le travail intellectuel et physique, celui déterminé par le sexe ; et il parle aussi de celui qui concerne les immigrants régulièrement racialisés. Sur les Roms, voir aussi : Balibar E., Foreword to Romani Politics, http://www.reseau-terra.eu/article1048.html, page consultée le 26 mai 2011. 
racial très profondément ancré qui n'est point nouveau (cette ancienneté est importante et pèse lourd), qui est transmis par la culture souvent « inconsciente » et des pratiques sociales non réfléchies : or, c'est exactement ce qui arrive aux dalit. En cela, l'exclusion des Roms de la visibilité publique acceptée ressemble à celle des femmes, plutôt qu'à d'autres groupes - elle porte une haute charge de symbolique. Et les Roms comme les femmes font le travail que les autres ne veulent pas faire. Ou en tout cas le faisaient, car les métiers traditionnels des Roms dépérissent et ils se retrouvent de plus en plus dans la catégorie des personnes « jetables », c'est-à-dire inexploitables.

La discrimination par naissance se pratique aussi dans d'autres cas. Il suffit de penser au «sang bleu », mythe dans ce cas soit positif, soit considéré comme inoffensif. Il évoque une distinction par l'origine quand il s'agit des familles royales ou aristocratiques et de leurs merveilleux mariages qui font rêver le Royaume-Uni, la Suède, etc.

Tout ceci est doublement intéressant : nous avons bien des mécanismes de répulsion et des comportements castéistes en Europe, mais nous ne le appelons pas ainsi et ne les reconnaissons pas; et nous faisons du concept de caste un modèle punitif, discriminatoire, normatif et raciste par lequel nous stigmatisons l'Inde comme une « société de castes ». Mais d'autres concepts que nous utilisons sont également normatifs, tels que celui de politique. En effet, la « caste » est considérée comme étant de l'ordre du social et non du politique. Nous sommes donc doublement excusés : s’il devait y avoir des castes en Europe, ce ne serait pas grave puisqu'elles relèveraient de la sphère du social, qu'une ruse des sciences sociales a séparée de celle du politique.

Bien sûr, il y a d'autres figures du dedans-dehors dans le limbe des confins, Ne qui ne altrove 6 , et la condition des Roms s'apparente par d'autres aspects à celles des migrants contemporains ou de certains autres groupes inclus en subordination : l'Europe devrait, pour dépasser ces partages qui se perpétuent, voir et surmonter son propre système des castes (ou son apartheid, selon Balibar ${ }^{7}$ ) ; celui-ci s'applique à ceux dont la condition est la pire depuis très longtemps, à ceux qui ne sont pas perçus comme humains tout simplement parce qu'ils ne sont même pas perçus. La liste en est longue et variable, mais est loin de s'épuiser. Pour le reconnaître, l'Europe devrait pouvoir assumer l'héritage historique au sein de la modernité qui la rend contra-

6. Mezzadra S. et Brett Neilson B., "Ne qui ne altrove - Migration, Detention, Desertion: A Dialogue”, Borderlands, e-journal, 2, 1 : www.borderlandsejournal.adelaide.edu.au/ issues/vol2no1.html, page consultée le 26 mai 2011. Une traduction italienne est parue dans la nouvelle édition de Diritto di fuga. Migrazioni, cittadinanza, globalizzazione, Vérone, Ombre corte 2001, entre-temps sortie augmentée par quelques textes, dont les entretiens avec Brett Neilson, Etienne Balibar et le Colectivo Situaciones à Buenos Aires (2006, même éditeur).

7. Balibar É., Nous citoyens d'Europe ? Les frontières, l'État, le peuple, Paris, la Découverte 2001. 
dictoire et partagée en elle-même ${ }^{8}$, l'histoire de la colonisation, la fin de la Guerre froide, la situation en Palestine-Israël qui fait partie de sa responsabilité historique, la condition des femmes, des Roms, etc. Elle s'est dotée d'hémisphères (est, ouest) inconscients qui n'ont pas été capables de s'intégrer à la construction de sa société (parce qu'inconscients et parce que sans lien entre eux), ainsi que d'une coupure d'avec l'Asie, le monde arabe et l'Afrique, avec lesquels elle est diversement en continuités territoriales et historiques.

Il n'y a pas plus, ni moins, de castes en Inde qu'en Europe. Il y a des sociétés différemment inégalitaires qui n'ont pas forcement de leçons à donner les unes aux autres. La société indienne est extrêmement, tragiquement, et aussi historiquement inégalitaire, sans aucun doute. Mais ce n'est pas d'elle dont il est question ici.

Stockholm, le 21 janvier 2011

8. Derrida J., L'antre cap, Paris, Minuit, 1991. 